\title{
Professionelle Handlungskompetenz von BNE-Akteuren
}

\author{
Verena Reinke
}

\section{Zusammenfassung}

Mit Beschluss des Global Action Programme on ESD (2015-2019) als Nachfolgeaktivität der UN-Dekade ist erneut die große Bedeutung der Lehrkräfte im Bereich BNE betont worden, denn effizienter Unterricht ist abhängig von der individuellen und spezifischen professionellen Handlungskompetenz, welche ein noch stark zu erforschendes Feld in der Fachdidaktik darstellt. Sowohl Lehrkräfte als auch nonformale Akteure lehren BNE und müssen über ein Repertoire an Kompetenzen verfügen, um Schüler auf globale Herausforderungen vorzubereiten.

Wie sich diese professionelle Kompetenz zusammensetzt, liegt im zentralen Erkenntnisinteresse des Projektes. Gemessen wird diese Kompetenz mit einem standardisierten Messinstrument, das basierend auf den Theorien zur professionellen Handlungskompetenz und Kompetenzkonzepten der BNE entwickelt wurde. Thematisch sind die Items überwiegend auf das exemplarische Thema „Klimawandel“ ausgerichtet, welches viele BNE-relevante Themenaspekte bietet und sowohl im schulischen als auch im außerschulischen Bildungsbereich thematisiert wird.

\section{$1 \quad$ Bildung für nachhaltige Entwicklung im Fach Geographie}

Mit dem Auslaufen der UN-Dekade im Jahr 2014 und dem Inkrafttreten des World Global Action Programme (Unesco 2013, WAP, 2015-2019) stehen Bildungsinstitutionen erneut an einem Punkt, an dem auf bisherige Erfolge im Bereich „Bildung für nachhaltige Entwicklung“ (BNE) zurückgeblickt werden kann, aber an dem auch

(C) Der/die Autor(en) 2017

K.-D. Altmeppen et al. (Hrsg.), Nachhaltigkeit in Umwelt,

Wirtschaft und Gesellschaft, DOI 10.1007/978-3-658-14439-5_10 
die noch zu erforschenden Felder zur Optimierung neu definiert werden sollten. In den vergangenen Jahren haben sich verschiedene Forschungsfragen bezüglich der Bildung für nachhaltige Entwicklung herauskristallisiert. Eine wichtige Rolle spielt in diesem Kontext das Professionswissen der Multiplikatoren, die zentral für die Implementierung einer BNE sind.

„Educators are one of the most important levers to foster educational change and to facilitate learning to sustainable development (UNESCO, 2013: 7)."

Dieses Zitat betont bereits die große Bedeutung von Lehrpersonen im Rahmen einer Bildung für nachhaltige Entwicklung, welche bereits in der UN-Dekade, aber nun auch mit Blick auf die Zukunft im Weltaktionsprogramm deutlich wird, in dem ihre Stärkung und Ausbildung zu den fünf wichtigsten Zielen gehören, was zugleich den großen Forschungsbedarf impliziert, um Antworten auf die Frage zu finden, was Lehrkräfte und nonformale Multiplikatoren eigentlich dazu befähigt, kompetent BNE in den Unterricht oder in außerschulische Bildungsveranstaltungen zu integrieren.

Das hier vorgestellte Projekt „Professionelle Handlungskompetenzen von BNE-Akteuren" beschäftigt sich konkret mit der Frage nach der vorhandenen Ausbildung der professionellen Handlungskompetenz von BNE-Multiplikatoren im schulischen und außerschulischen Bereich und will diese mit einem standardisierten Fragebogen erheben, um anhand der Ergebnisse erste Rückschlüsse auf bereits verfügbare Ressourcen ziehen und so ein empirisch gestütztes Profil professioneller Handlungskompetenz beschreiben zu können.

Bildung für nachhaltige Entwicklung ist in den Bildungsstandards für die Geographie als besondere Bildungsaufgabe hervorgehoben, darüber hinaus wird diesem Fach im Zuge der Rückumstellung auf G9 in Niedersachsen eine steuernde Funktion für BNE im Kerncurriculum zugeschrieben, was die Relevanz der Geographie im Rahmen der langfristigen Implementierung in der Schule deutlich macht. Was jedoch genau unter diesem besonderen Bildungsauftrag zu verstehen ist, mag auch nach Abschluss der UN-Dekade nicht jeder Geographielehrkraft bekannt sein, sodass davon auszugehen ist, dass zwar Bildung für nachhaltige Entwicklung jedem ein Begriff ist, das konzeptionelle Leitbild der Nachhaltigkeit und die damit verbundenen Intentionen von BNE jedoch nicht. Dies könnte dazu führen, dass Lehrkräfte BNE als Zusatzaufgabe empfinden und weniger als Leitbild, an deren Idealen sie die Gestaltung ihres Unterrichts orientieren können. Durch die hohe konzeptionelle Affinität des Faches Geographie mit BNE (Bagoly-Simó, 2014) ist jedoch davon auszugehen, dass Geographielehrkräfte bereits eine gute 
Grundvoraussetzung mitbringen, um BNE in den Unterricht zu integrieren, ohne dass diese als Zusatzbelastung empfunden wird.

Das integrative Kompetenzkonzept für BNE (de Haan, 2008) weist zudem in der Operationalisierung der Gestaltungskompetenz viele Ähnlichkeiten zu den zu fördernden Kompetenzen des Schulfaches Geographie auf. Damit kann man davon ausgehen, dass Schülerinnen und Schüler im Rahmen des Geographieunterrichts Unterstützung beim Erlangen der Gestaltungskompetenz bekommen. Allerdings geht es bei BNE nicht nur um Kompetenzen. Diese werden schließlich an BNE-Inhalten erlernt, eine effektive BNE kommt nicht ohne inhaltliche Anbindung aus. Das Fach Geographie behandelt empirischen Studien zufolge eine große Zahl an BNE-Inhalten und kann damit als Hauptträgerfach einer BNE verstanden werden (z. B. Bagoly-Simó, 2014).

\section{Forschungsanlass und zentrale Fragestellung}

Während die relevanten BNE-Kompetenzen für Lerner durch die Gestaltungskompetenz (de Haan et al., 2008: 183f.) bereits beschrieben und in Teilkompetenzen gegliedert sind, also ein Kompetenzmodell existiert, gibt es für die Multiplikatoren bisher kaum Erkenntnisse, die sich auf die professionelle Handlungskompetenz von Lehrkräften oder außerschulischen Multiplikatoren im Bereich BNE beziehen. Die wenigen Modelle, die entwickelt wurden, richten sich eher auf soziale Kompetenzen und das Arbeiten im Team (Steiner, 2011) als auf kognitive Kompetenzen (Hellberg-Rode, Schrüfer \& Hemmer, 2014). Dies ist eine Herausforderung, die den Forschungsbedarf auch dadurch deutlich macht, dass der Pool an BNE-Akteuren sehr breit gefächert ist. So gehören dazu sowohl Lehrkräfte als auch außerschulische Multiplikatoren in Umweltzentren oder sogenannten Weltläden, die sich mit BNE beschäftigen und diese mit großer Motivation in ihren Bildungsveranstaltungen praktizieren. All diese Multiplikatoren agieren auf einer unterschiedlichen Kompetenzbasis, die wiederum auf verschiedenen Expertisen beruht, was durch die Wahl der Studienfächer oder Berufsausbildungen begründet ist. Bezüglich der schulischen BNE-Akteure ist der Ausbildungshintergrund im Vorfeld transparenter: Jede Lehrkraft hat ein Studium absolviert; so kann davon ausgegangen werden, dass diese Probanden fachwissenschaftliche und pädagogische Grundkenntnisse haben. Darüber hinaus verfügen sie über eine fachdidaktische Ausbildung in ihren Fächern. Ob sie im Rahmen des Studiums jedoch auch Kenntnisse im Bereich BNE bekommen haben, unterscheidet sich vermutlich sehr stark. BNE als fachübergreifendes 
Bildungskonzept sollte eigentlich in verschiedenen Fachdisziplinen thematisiert und nicht isoliert betrachtet werden.

BNE intendiert, bei den Lernenden eine Gestaltungskompetenz zu fördern, um heutige Lebensstile der Gesellschaft beurteilen, nicht nachhaltiges Agieren erkennen und vermeiden sowie nachhaltige Handlungsalternativen aufzeigen zu können. Die Gestaltungskompetenz ist über zwölf Teilkompetenzen näher differenziert (de Haan et al., 2008). Inwiefern jedoch deren Aufbau bzw. die Förderung dieser gelingt, ist maßgeblich abhängig von erfolgreicher Bildungsarbeit. Somit ist die Wirksamkeit der BNE an die Ausprägung der spezifischen professionellen Handlungskompetenz dieser Multiplikatoren gebunden. Für den Fachunterricht ist die Bedeutung des kognitiven Fachwissens und fachdidaktischen Wissens bereits durch empirische Studien nachgewiesen (Kunter et al., 2011), die auf den einschlägigen Theorien zur professionellen Handlungskompetenz basieren.

Diese umfassen jedoch noch weitere, nämlich motivationale, Aspekte, deren Bedeutung für die Erstellung eines Profils von professioneller Handlungskompetenz von BNE-Akteuren noch erfasst werden muss. Dies gilt nicht nur für Lehrkräfte, sondern ebenso für die zahlreichen nonformalen Multiplikatoren, die im Bereich BNE, z. B. in Umweltzentren oder Eine-Welt-Zentren, agieren und mit großem Engagement tätig sind. Gerade bei dieser Akteursgruppe ist der berufliche Hintergrund sehr vielfältig; die Multiplikatoren kommen aus unterschiedlichen Fachrichtungen, bringen jedoch vermutlich ein ausgeprägtes Interesse explizit an nachhaltigkeitsrelevanten Themen mit und arbeiten nach den Zielen der BNE. Diese zielt darauf ab, Wissen über nachhaltige und nicht nachhaltige Prozesse zu vermitteln und die Analyse- und Reflexionsfähigkeit zu fördern, womit BNE mehr fordert als rein fachspezifische Kompetenzen. Auch Fächer mit besonders hoher Relevanz für BNE wie die Geographie, die in diesem Projekt im Fokus steht, können diesbezüglich nicht isoliert betrachtet werden. Ein pädagogischer Hintergrund für BNE, der auf einer Ausbildung beruht, ist nicht automatisch gegeben.

Sowohl für Geographie-Lehrkräfte als auch für die außerschulischen Multiplikatoren sind die fachwissenschaftlichen und fachdidaktischen Facetten des Professionswissens bisher nicht erfasst; beide Gruppen sollten aber über Wissen bzw. Kompetenzen verfügen, um wirksame Bildung durchführen zu können.

Empirische Befunde zu der Bedeutung der anderen Komponenten, die zur professionellen Handlungskompetenz gehören, fehlen. Dazu werden die motivationalen und sozialen Faktoren gezählt, über die bisher jedoch wenig bekannt ist. Nicht nur in dem oben zitierten World Action Programme for Sustainable Development wird die Bedeutung der Lehrperson deutlich, auch die in der Bildungslandschaft viel diskutierte Studie John Hatties (2014) stellt heraus, dass es eben nicht gleichgültig 
ist, wer vor der Klasse steht und dass vor allem das Handeln der Lehrperson einen großen Einfluss hat.

Aus den hier dargelegten Grundlagen und Problemstellungen ergibt sich die zentrale Forschungsfrage des Projektes:

Über welche professionelle Handlungskompetenz verfügen BNE-Multiplikatoren, zu denen sowohl Lehrkräfte als auch nonformale Akteure gehören, und welche Unterschiede und Gemeinsamkeiten ergeben sich zwischen diesen beiden Akteursgruppen?

Im Rahmen der Studie wird über eine quantitative Befragung mit einem standardisierten Fragebogen bei schulischen und nonformalen BNE-Akteuren erhoben, welchen Anteil die jeweiligen Komponenten der professionellen Handlungskompetenz bei den Lehrkräften und bei nonformalen Akteuren haben, sodass Unterschiede bzw. Gemeinsamkeiten dargelegt werden können. Die Ergebnisse sollen eine Grundlage für ein empirisch-gestütztes Profil professioneller Handlungskompetenz von BNE-Akteuren bilden, an denen sich die künftige Ausbildung von Lehrkräften und Multiplikatoren orientieren kann.

\section{$3 \quad$ Theoretischer Hintergrund und Herleitung des Modells}

Die professionelle Handlungskompetenz ist bisher auch für Lehrkräfte nur in wenigen Studien erfasst worden, die bekannteste hierzu ist die aus der Fachdidaktik Mathematik stammende COACTIV-Studie (Kunter et al., 2011). In den Fachdidaktiken hat sich bisher eine Einteilung von Facetten professioneller Handlungskompetenz durchgesetzt, die auf Lee Shulman (1986) zurückgeht, was auch für das Modell der COACTIV-Studie zutrifft. Über die Einteilung von Shulman herrscht weitgehend Konsens: Sie differenziert in general pedagogical knowledge, subject matter content knowledge, pedagogical content knowledge, curriculum knowledge. Diese Unterscheidung erweitert Shulman um weitere Bereiche: Psychologie des Lerners, Organisationswissen und bildungstheoretisches und -historisches Wissen (Kunter et al., 2011: 33f.).

Das erwähnte Modell ist zur Messung von Kompetenzen im Fachbereich Mathematik entwickelt worden, somit also auf eine Fachdomäne bezogen und nicht ohne Modifizierung auf ein anderes Fach übertragbar. Das Messmodell arbeitet mit Facetten, die sich auf einzelne Komponenten der professionellen Handlungskompetenz beziehen. Diese Systematisierung in kognitive sowie nicht-kognitive Kompetenzfacetten kann auch für ein Messmodell im Bereich Bildung für nach- 
haltige Entwicklung übernommen werden. So wurde auf dieser Grundlage ein eigenes Modell entworfen.

Das für die vorliegende Studie entworfene Kompetenzmodell (s. Abbildung) wurde entsprechend der Probanden modifiziert, da beispielsweise wegen der rein schulischen Fokussierung des COACTIV-Modells eine leichte Abänderung der Subfacetten im Hinblick auf die außerschulischen Akteure notwendig war.

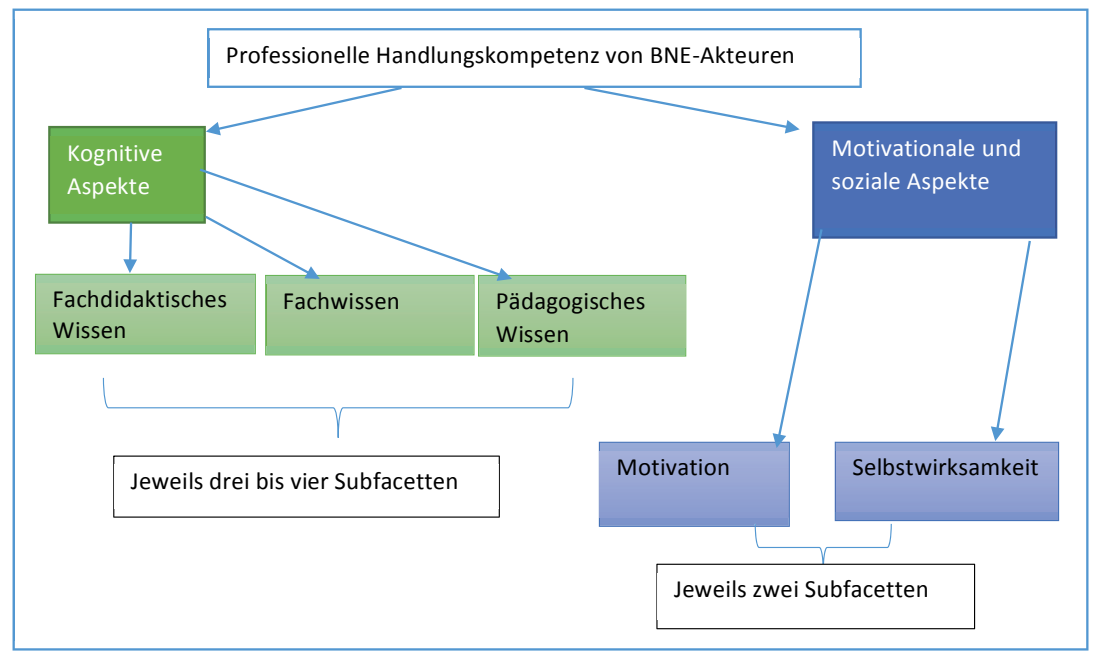

Abb. Kompetenzbasismodell zur Konstruktion des Erhebungsinstruments Quellenangabe: Eigener Entwurf in Anlehnung an Kunter et al. 2011: 32.

Die im Modell aufgeführten Facetten sind weiter unterteilt in Unterpunkte (Kunter et al., 2011: 32), die auf die Struktur des Bildungskonzeptes „Bildung für nachhaltige Entwicklung" abgestimmt wurden. So wird bei der Unterfacette „Fachwissen“ nicht lediglich fachspezifisches Wissen der Geographie abgefragt, sondern es wird hier dem fachübergreifenden Bildungskonzept Rechnung getragen. Die Fokussierung auf das Fach Geographie begründet zwar eine inhaltliche Orientierung an einem geographischen Sachverhalt, jedoch wurde bei der Konzeption des Erhebungsinstruments darauf geachtet, ein Thema zu wählen, das gut fachübergreifend thematisiert werden kann. Das so abgewandelte Modell wurde als Grundlage für das Messinstrument zur Erfassung der professionellen Handlungskompetenz der BNE-Akteure genutzt. 
Ein übergeordnetes Forschungsanliegen des vorliegenden Projektes ähnelt daher der Intention von COACTIV, „[...] die individuellen Merkmale zu identifizieren, die Lehrkräfte für die erfolgreiche Bewältigung ihrer beruflichen Aufgabe benötigen (Kunter et al., 2011: 29).“ Im vorliegenden Projekt ist dieses Anliegen um die Komponente der nonformalen Multiplikatoren erweitert, die für ihre Tätigkeit ebenso auf ein Repertoire an professioneller Handlungskompetenz zurückgreifen müssen.

Einbezogen in die Erstellung des Modells ist zudem eine Studie aus der Politikdidaktik, die sich mit der professionellen Kompetenz von Politiklehrkräften beschäftigt (Weschenfelder, 2014) und als Basis ebenfalls auf die Shulman-Theorie zurückgreift.

Weiterhin dient die Expertiseforschung im Lehramt (Bromme, 1992) als weitere Grundlage für die Strukturierung der Subfacetten, die für das konzipierte Kompetenzmodell gebildet wurden. So finden hierbei unterschiedliche Formen des Wissens Berücksichtigung: Überwiegend beziehen sich die Items zur Testung des Wissens auf den theoretisch-formalen Wissenstyp, zu dem primär das Fachwissen von Lehrkräften zählt, daneben wurden aber auch Items aus dem fachdidaktischen und pädagogischen Wissensbereich formuliert (Kunter et al., 2011: 35). Letzterer jedoch weist auch praktische Anteile auf.

\section{$4 \quad$ Konzeption des Messinstruments}

Das Erhebungsinstrument (standardisierter Fragebogen) beinhaltet Aufgabenstämme, an die sich offene oder geschlossene Fragen anschließen. Diese Items sind den in der Abbildung erwähnten Subfacetten zugeordnet, die in den folgenden Unterkapiteln erläutert werden. Als übergeordnetes Thema für die fiktiven Beispiele ist das Thema Klimawandel gewählt worden. Sowohl der Geographieunterricht als auch Bildungseinrichtungen, die sich primär dem Globalen Lernen als Teilbereich der BNE widmen und auch die, die einen eher ökologischen Schwerpunkt haben, beschäftigen sich im Rahmen ihrer Tätigkeit mit dem Klimawandel, sodass mit diesem Oberthema hier eine gemeinsame Basis gefunden wurde.

\subsection{Fachwissen in der Bildung für nachhaltige Entwicklung}

In diesem Projekt wird das Fachwissen für BNE zwar aus der geographiedidaktischen Forscherperspektive betrachtet, jedoch ist bei der Modellierung der Items bedacht worden, dass sich die Bildung für nachhaltige Entwicklung aus verschiedenen 
Fachdisziplinen zusammensetzt. Die Items beziehen sich inhaltlich auf Ursachen, Folgen und Maßnahmen bezüglich des Klimawandels. Eine Einteilung, die typisch für die Geographie, aber auch typisch für die Analyse der großen Umwelt- und Entwicklungsprobleme ist.

Für das Fachwissen wurden folgende Subfacetten hergleitet:

1. Allgemeines Wissen eines Erwachsenen,

2. Forschungswissen und

3. Wissen über Konzepte und Strukturen der Nachhaltigkeit.

Dieser Differenzierung von Wissen liegen verschiedene Studien zugrunde, so z. B. die Forschungen von Seeber \& Minnameier (2010). Maßgeblich war es, Items zu entwickeln, die inhaltlich sowohl das Tätigkeitsfeld der Lehrkräfte als auch der außerschulischen Akteure abdecken, somit musste ein gemeinsamer Bezugsrahmen gegeben sein.

Für die Bestimmung eines normativen Bezugsrahmens sind neben den generell zu unterscheidenden Anspruchsniveaus unterschiedliche konzeptionelle Leit- und Gestaltungsideen, die den curricularen Anforderungen auf universitärem und auf berufsschulischem Niveau zugrunde gelegt wurden, zu berücksichtigen (Seeber \& Minnameier, 2010: 128). Was die Autoren hier für kaufmännische Berufe feststellen, ist auch für die Bildung für nachhaltige Entwicklung ein sinnvoller Hinweis, gerade weil sie in ihrer Komplexität von vielen verschiedenen Multiplikatoren mit unterschiedlicher Expertise praktiziert wird. BNE ist nach dem Begriffsverständnis von Gruber \& Mandl eine „schlecht definierte Domäne“ (2006, zit. nach Seifried \& Ziegler, 2009: 86), die sich durch Komplexität und verschiedene Lösungswege auszeichnet. Die Durchdringung der Fachstruktur ist aber maßgeblich dafür, dass das Fach vermittelt werden kann.

Shulman beklagt 1986, dass in vorherigen Tests in den USA der Übergang des Inhalts in die Vermittlung nicht bedacht worden sei und somit die Bedeutung des Inhalts fehle. Er definiert „content knowledge“ als Summe und Organisation von Wissen, bei der verschiedene Wege des Strukturierens verwendet werden. Der Lehrer müsse nicht nur verstehen, dass etwas so ist, sondern auch, warum es so ist. Der Autor weist dem Lehrkörper eine besondere Verantwortung zu, da er für den Schüler in der Regel die erste Wissensquelle ist (Shulman, 1986: 9). Dies bedeutet, die Struktur des Fachwissens zu erfassen, was in diesem Fall ausgeweitet werden muss auf das Wissen mehrerer Fächer, die für das BNE-Wissen und den Klimawandel relevant sind.

Schon bei einem konkreten Fach ist von einer hohen Domänenspezifität auszugehen, handelt es sich aber um eine komplexe Domäne, die viele Wissensbereiche 
aus unterschiedlichen Fachdisziplinen erfasst, dann muss der Multiplikator über Wissen verfügen, sich das Notwendige zu erschließen, da hohes Wissen auf einem Gebiet nicht automatisch hohes Wissen in einem anderen bedeutet (Tepner et al., 2012: 9). Eine Befragung zum spezifischen BNE-Professionswissen von Lehrkräften zeigte bereits, dass Themenzuordnungen und Relevanzen von Einzelthemen für BNE sehr unterschiedlich ausgefallen sind (Hellberg-Rode, Hemmer \& Schrüfer, 2014).

Um das bei den Probanden vorhandene Fachwissen zum Thema besser differenzieren zu können, wurden die zuvor benannten Facetten in unterschiedliche Subfacetten eingestuft. Wie in der ProwiN-Studie herausgestellt wird, gilt auch für die BNE-Multiplikatoren, dass neben dem allgemeinen Wissen eines Erwachsenen, Wissen vertieft vorhanden sein muss und über die Schulstufe hinausgehen soll (Tepner et al., 2012: 10). Es sind in Bezug auf BNE jedoch noch einige Ergänzungen notwendig. So sollten die Multiplikatoren in jedem Fall Wissen über das Konstrukt der Nachhaltigkeit besitzen, was auch in der oben zitierten Studie (Hellberg-Rode, Hemmer \& Schrüfer, 2014) von den befragten Experten als wichtig erachtet wird.

Um Fachwissen abzufragen, das über das allgemeine Wissen eines Erwachsenen hinausgeht, fragen die Items zum „Forschungswissen“ nach vertieften Kenntnissen, um zu prüfen, ob die Zusammenhänge bekannt sind, im Erhebungsbogen 2015 zum Beispiel: „Nennen Sie Ihnen bekannte Rückkoppelungseffekte beim Klimawandel.“

Hier wird anhand der gegebenen richtigen Antworten getestet, welches vertiefte Fachwissen die Probanden zu diesen Phänomenen haben, und bei der Auswertung zwischen den Ergebnissen der Akteursgruppen verglichen. Dieses Item wurde gewählt, um zu prüfen, ob die Probanden über vertieftes Wissen über Zusammenhänge verfügen. Dies ist notwendig, um Inhalte wirksam zu vermitteln.

\subsection{Fachdidaktisches Wissen in der Bildung für nachhaltige Entwicklung}

Die Geographie als Trägerfach mit ihrer hohen Affinität zu BNE ist auch für das fachdidaktische Wissen in besonderem Maße relevant. Lehrkräfte mit dem Fach Geographie sind durch eben diese Affinität bereits durch ihre Ausbildung auf die didaktische Aufbereitung von Mensch-Umwelt-Thematiken, wie z. B. Klimawandel, vorbereitet, sie dürften so prädestiniert sein für die Integration von BNE in den Unterricht.

Diese Thematisierung von Mensch-Umwelt-Themen an der Schnittstelle zwischen Natur- und Gesellschaftswissenschaften sind gute Grundvoraussetzungen, um ein BNE-relevantes Thema in den Unterricht zu integrieren. Der Klimawandel mit seiner Komplexität und der Notwendigkeit von Wissen aus dem naturwissenschaftlichen 
und gesellschaftswissenschaftlichen Bereich bietet sich als exemplarisches Thema für BNE hier an.

Daneben existiert als Bezugsrahmen für das fachdidaktische Wissen die Gestaltungskompetenz, die es bei den Lernern zu fördern gilt. BNE-Akteure lehren nicht nur spezifische Lehrinhalte, sondern gerade in Bezug auf die Gestaltungskompetenz ist aus fachdidaktischer Sicht einzubeziehen, wer die Lernenden sind (Adressaten), in welchem Rahmen agiert wird und welche Teilkompetenzen dabei besonders fokussiert werden (Seifried \& Ziegler, 2009: 85). Diese Aspekte korrelieren vermutlich auch mit der Motivation, die mit der Domäne in Zusammenhang steht, so z. B. mit dem Enthusiasmus für BNE (-Inhalte) bzw. den Klimawandel.

Der Domänenbegriff ist in Anlehnung an Bromme (1992) im Hinblick auf BNE interessant, da er von einer "Philosophie des Schulfaches“ (zit. nach Seifried \& Ziegler, 2009: 86) spricht, was bei Seifried \& Ziegler als „Nützlichkeit eines Lerninhaltes und dessen Beziehungen zu anderen Lebens- und Wissensbereichen “ (Seifried \& Ziegler, 2009: 86) beschrieben ist. Letzteres deutet bereits auf ein wesentliches Charakteristikum der BNE hin, die mit ihrem interdisziplinären Charakter vernetztes Denken von allen Beteiligten fordert, das in kompetente Handlung überführt werden soll. Für Lehrkräfte und Multiplikatoren im außerschulischen Bildungsbereich bedeutet dies aber auch, dass sie zunächst die Struktur des Inhalts so durchdringen müssen, dass sie in der Lage sind, ihn zu lehren und über ein Repertoire an Erklärungsmöglichkeiten verfügen müssen.

$\mathrm{Zu}$ dem fachdidaktischen Wissen zählt auch die Fähigkeit, Lernerkognitionen nachvollziehen zu können, um den Lernern einen Unterrichtsgegenstand auf verschiedene Arten erklären zu können.

Es ergeben sich folglich für das fachdidaktische Wissen folgende Subfacetten:

1. Erklären, Repräsentieren, Skizzieren (Vermittlung des Inhaltes),

2. Handlungsorientierung der Lerner, Schulung der Meinungskompetenz (Vermittlung von Handlungskompetenz, Bewertungskompetenz),

3. Lernerkognition, Schulerfehler, Lernumgebung (Einflussfaktoren beim Verständnis des Stoffes von den Lernern, Verstehen von auseren Einflussfaktoren auf den Lernprozess) und

4. Aufgaben, multiples Lösungspotenzial (Fähigkeit, verschiedene Aufgaben zur Durchdringung eines Inhaltes zu stellen).

Diesen Facetten sind jeweils drei bis vier Items zugeordnet, die ein offenes oder geschlossenes Antwortformat haben. Im Folgenden ein Beispiel für ein offenes Itemformat aus dem Erhebungsbogen 2015: Item der Subfacette „Erklären, Repräsentieren, Skizzieren“: 
Eine Schülerin zeigt auf. Sie hat in einem Schulbuch den Begriff „anthropogen“ gelesen und fragt, was er bedeute, viele andere Schüler wissen es auch nicht. Bitten geben Sie an, wie Sie den Begriff an dieser Stelle in das Unterrichtsgeschehen einbinden würden.

Ein entwickeltes Kategoriensystem ermöglicht die Auswertung der Beschreibungen, sodass aus den Antworten heraus die relevanten Elemente zugeordnet werden können.

\subsection{Pädagogisches Wissen}

Diese Komponente des kognitiven Professionswissens ist unabhängig von einer Fachdomäne zu betrachten, sodass hier z. B. auch auf Itemkonstruktionen aus der COACTIV-Studie zurückgegriffen werden konnte. Dabei galt es jedoch zu beachten, dass bei den Probanden dieser Studie nicht zwangsläufig alle ein Studium absolviert haben, wobei dies bei den Lehrkräften der Fall ist. Daher wurden auch hier Modifizierungen der Subfacetten vorgenommen, sodass sich folgende Differenzierung ergab:

1. Wissen über Organisation von Lernsituationen,

2. Wissen über theoretische Konzepte und Lernprozesse und

3. Methoden.

Die Herleitung erfolgte auf Grundlage der Studien von Shulman (1986), Kunter et al. (2011), Bonsen \& Priebe (2013) sowie Schrittesser (2011).

Betrachtet man die Bedeutung des Terminus „Pädagogik“, die Theorie und Praxis der Erziehung und Bildung, so wird bereits ein häufig beschriebener Konflikt deutlich: Theorie und Praxis - wie gelingt es dem Lehrer letztlich, die im Studium gelernte Theorie auch in der Praxis anzuwenden oder agiert er dann doch eher aus einer erworbenen Routine heraus? Gerade im Hinblick auf die vorliegende Studie zum Professionswissen von BNE-Akteuren, zu denen sowohl Lehrkräfte als auch nonformale Akteure zählen, sollte bedacht werden, dass unter den nonformalen Kräften einige Personen sein könnten, die keine pädagogische Ausbildung haben, dennoch aber aus ihrer Sicht pädagogisch arbeiten und durchaus gut strukturierte Workshops anbieten. Es ist gleichzeitig zu bedenken, dass es sicher auch viele Erziehungswissenschaftler gibt, die dort arbeiten. Die Fachliteratur zum Thema „Pädagogisches Wissen“ lässt erkennen, dass auch dieser Aspekt der professionel- 
len Handlungskompetenz schwer zu definieren ist. Zudem ist er in der bisherigen Forschung fast ausschließlich in Bezug auf die Lehrkräfte diskutiert worden, was die Übertragung der Kenntnisse auf außerschulische Akteure erschwert.

Ein „pädagogisches Berufsethos“ (Bonsen \& Priebe, 2013: 4) gilt eben nicht nur für die Lehrkräfte, sondern sollte auch andere, am Bildungsprozess beteiligte Personen, einbeziehen. Die genannten Autoren widersprechen hier dem Lehrerethos und der alleinigen pädagogischen Verantwortung der Lehrer. Dieser Ansatz ist daher auch auf Multiplikatoren und den Gesamtzusammenhang übertragbar und maßgeblich bei der Konzeption der Items gewesen.

Sowohl die schulischen als auch die außerschulischen Probanden sollten über die Fähigkeiten verfügen, Inhalte zu vermitteln. Die „Vermittlungskompetenz“ (Schrittesser, 2011: 102) steht im Vordergrund als Kernkompetenz.

Ein weiteres Phänomen des Pädagogischen liegt darin, dass pädagogisches Handeln häufig in Situationen gefordert ist, die nicht planbar sind. Somit ist es nicht möglich, einer Programmatik oder einem bestimmten Anwendungsschema $\mathrm{zu}$ folgen. Wimmer beschreibt sogar das Nicht-Wissen als Kern des pädagogischen Handelns (zit. nach Schrittesser, 2011: 103).

Dies zeigt das Item der Subfacette „Wissen über Organisation von Lernsituationen“ aus dem Erhebungsbogen 2015 (Beispiel angelehnt an Kunter et al., 2011).

\section{Fallbeispiel 3: Unterrichtsstörungen}

Die Schüler vertiefen gerade ein Thema und sind mit einer Aufgabe beschäftigt. In der Besprechung dieser wird angeregt diskutiert. Sie sind größtenteils bei der Sache. Timo jedoch nicht, er ruft öfter etwas hinein, was nicht zum Thema gehört. Er beginnt in der Tasche zu kramen, Sie glauben, dass er in der Tasche auf ein Smartphone schaut.

Was würden Sie tun? Bitte kreuzen Sie an (Einfachnennung):

o Unauffällig nähern und beobachten.

o Vor der Klasse den Jungen ansprechen und ihn ermahnen.

o Fragen, was er da mache.

o Timo nach draußen schicken.

o Der Klasse eine Aufgabe geben und Timo dann ansprechen.

Dieses Beispiel mit geschlossenem Antwortformat zielt auf die unterschiedlichen Handlungsstrategien der Lehrenden ab. Daran schließen sich weitere Items mit offenem Antwortformat an, die sich ebenfalls auf die geschilderte Unterrichtsstörung beziehen. 


\section{Durchführung der Befragung und Stichprobe}

Die Erhebung wurde in zwei Phasen durchgeführt. Von Februar bis Juni 2014 fand eine Vorstudie statt. Ziel dieser war es, exemplarisch über einen qualitativen Zugang die Tätigkeit der befragten Bildungsakteure konkreter fassen zu können, da diese in der Regel über sehr unterschiedliche Expertisen verfügen, wobei diese erste Erhebung als Vorstudie zur Hypothesengenerierung anzusehen ist.

Es wurden leitfadengestützte Interviews mit fünf nonformalen Bildungsakteuren geführt. Der Leitfaden bezieht sich auf die einzelnen Facetten, die sich auch im Haupterhebungsinstrument finden. Ferner wurden Fragen zu den Erfahrungen der Interviewpartner im Hinblick auf die Zusammenarbeit mit Schulen und mögliche Stärken und Schwächen dieser gestellt, um Rückschlüsse auf die Komponenten ziehen zu können. Die Dauer der Interviews variierte zwischen 60 und 80 Minuten. Die Interviews sind zur Hypothesengenerierung genutzt worden.

Nach Auswertung der Interviews sind die Hypothesen entsprechend modifiziert und die Haupterhebung vorbereitet worden. Die quantitative Hauptstudie erfolgte mit einem standardisierten Fragebogen, der sowohl Lehrkräften der Geographie als auch nonformalen Kräften vorgelegt wurde. Die Stichprobe umfasste 50 Geographielehrkräfte an niedersächsischen Gymnasien und 50 außerschulische Akteure, die in Umweltbildungszentren bzw. im Globalen Lernen tätig waren.

Aufgrund der bundesweit unterschiedlichen schulischen Organisation und der differenten Lehrpläne erfolgte im Hinblick auf die Lehrkräfte eine Beschränkung auf das Bundesland Niedersachsen, um z. B. als Grundlage des Unterrichtens den gleichen Lehrplan sowie die Stundentafel zu haben. Da bei den außerschulischen Akteuren eine solche gemeinsame Basis nicht gegeben ist, konnte hier eine Ausweitung über Niedersachsen hinaus gemacht werden, die auch erforderlich war, weil die Anzahl der außerschulischen Akteure weitaus geringer ist als die der Lehrkräfte.

Bei der Auswahl der Probanden wurde darauf geachtet, einen möglichst vielfältigen Pool zu haben, was sowohl die inhaltlichen als auch die regionalen Schwerpunkte betrifft. Ferner sind im Vorfeld die Schulprogramme bzw. Bildungskonzepte der außerschulischen Einrichtungen gesichtet worden, um ein repräsentatives Ergebnis erzielen zu können. Die quantitative Haupterhebung lief von Februar 2015 bis Juli 2016, die schriftliche Befragung wurde im paper-pencil-Verfahren während meiner Anwesenheit durchgeführt.

Die zuvor beschriebenen Wissensfacetten wurden mit einem standardisierten Fragebogen erhoben, der insgesamt 31 Items umfasst. Die Beantwortung des Bogens dauerte im Schnitt ca. 45 Minuten. 


\section{Ausblick}

Die Ergebnisse der Erhebungen werden derzeit ausgewertet. Dafür ist ein Kategoriensystem entwickelt worden, in das die Antworten der Probanden eingeordnet werden. Dieser Vorgang wird von zwei Ratern durchgeführt; im Anschluss daran werden die Ergebnisse statistisch ausgewertet und verschriftlicht. Die statistische Auswertung wird über Varianzanalysen erfolgen.

Die Messung der professionellen Handlungskompetenz von BNE-Akteuren soll Aufschluss geben über die bereits vorhandene Kompetenz und über den Vergleich der beiden Akteursgruppen und damit auch wichtige Hinweise darauf liefern, wie voneinander profitiert werden kann, um zukünftig die Aus- und Fortbildung von Multiplikatoren besser gestalten zu können.

Diese empirische Datengrundlage kann ferner eine wichtige Basis sein für weitere Forschungen im Bereich BNE, die z.B. die Wirksamkeit von Aus- und Fortbildungsinterventionen analysieren können.

\section{Literatur}

Bagoly-Simó, P. (2014). Implementierung von BNE am Ende der UN-Dekade. Eine internationale Vergleichsstudie am Beispiel des Fachunterrichts. Zeitschrift für Geographiedidaktik, 4: 221-256.

Bromme, R. (1992). Der Lehrer als Experte. Zur Psychologie des professionellen Wissens. Bern u. a.: Hans Huber.

Bonsen, M. \& Priebe, B. (2013). Unterwegs zu einem Berufsethos in der Schule. Zur Professionalität gehört eine pädagogische Berufsethik. Lernende Schule, 62: 4-7.

De Haan, G., Kamp, G., Lerch, A., Martignon, L., Müller-Christ, G. \& Nutzinger, H.-G. (2008). Nachhaltigkeit und Gerechtigkeit. Grundlagen und schulpraktische Konsequenzen. Berlin, Heidelberg: Springer.

Hattie, J. (2014). Lernen sichtbar machen für Lehrpersonen. Überarbeitete deutschsprachige Ausgabe von „Visible Learning for Teachers“. Hohengehren: Schneider Verlag.

Hellberg-Rode, G., Schrüfer, G. \& Hemmer, M. (2014). Brauchen Lehrkräfte für die Umsetzung von Bildung für nachhaltige Entwicklung (BNE) spezifische professionelle Handlungskompetenz? Theoretische Grundlagen, Forschungsdesign und erste Ergebnisse. Zeitschrift für Geographiedidaktik, 4: 257-281.

Kunter, M., Baumert, J., Blum, W., Klusmann, U., Krauss, S. \& Neubrand, M. (2011). Professionelle Kompetenz von Lehrkräften. Ergebnisse des Forschungsprogramms COACTIV. Münster u. a.: Waxmann.

Seeber, S. \& Minnameier, G. (2010). Zur Erfassung von fachlichem und fachdidaktischem Wissen von Lehrenden im Bereich der kaufmännischen Ausbildung. In: K. Beck \& O. 
Zlatkin-Troitschanskaia (Hrsg.), Lehrprofessionalität. Was wir wissen und was wir wissen müssen. Landau/Pfalz: Verlag Empirische Pädagogik (=Lehrerbildung auf dem Prüfstand, Jg. 3, Sonderheft).

Seifried, J. \& Ziegler, B. (2009). Domänenbezogene Professionalität. In: O. Zlatkin-Troitschanskaia, K. Beck, D. Sembill, R. Nickolaus \& R. Mulder (Hrsg.), Lehrprofessionalität. Bedingungen, Genese, Wirkungen und ihre Messung (pp. 83-92). Weinheim und Basel: Beltz.

Schrittesser, I. (2011). Professionelle Kompetenzen: Systematische und empirische Annäherungen. In: M. Schratz, A. Paseka \& I. Schrittesser (Hrsg.), Pädagogische Professionalität. Impuls für next practice im Lehrerberuf. Wien: Facultas.

Shulman, L.S. (1986). Those who understand: Knowledge growth in teaching. Educational Resarcher, 15(2): 4-14.

Steiner, R. (2011). Kompetenzorientierte Lehrer/innenbildung für Bildung für Nachhaltige Entwicklung. Kompetenzmodell, Fallstudien und Empfehlungen. Münster Verlagshaus Monsenstein und Vannerdat (= Schriftenreihe Bildung \& Nachhaltige Entwicklung).

Tepner, O. (2012). Modell zur Entwicklung von Testitems zur Erfassung des Professionswissens von Lehrkräften in den Naturwissenschaften. Zeitschrift für Didaktik der Naturwissenschaften, Jg. 18: 7-28.

Weschenfelder, E. (2014). Professionelle Kompetenz von Politiklehrkräften. Eine Studie zu Wissen und Überzeugungen. Berlin, Heidelberg: Springer.

UNESCO (2013): Proposal for a Global World Action Program on Education for sustainable development as follow-up to the United Nations Decade of Education for Sustainable Development (DESD) after 2014. http://unesdoc.unesco.org/images/0022/002243/224368e. pdf (zugegriffen 04.03.2016).

Open Access Dieses Kapitel wird unter der Creative Commons Namensnennung - Nicht kommerziell 2.5 International Lizenz (http://creativecommons.org/licenses/by-nc/2.5/deed. de) veröffentlicht, welche für nicht kommerzielle Zwecke die Nutzung, Verbreitung und Wiedergabe in jeglichem Medium und Format erlaubt, sofern Sie den/die ursprünglichen Autor(en), den Titel des Werks und die Quelle ordnungsgemäß nennen, einen Link zur Creative Commons Lizenz beifügen und im Falle einer Abwandlung durch einen entsprechenden Hinweis deutlich erkennbar machen, dass Änderungen vorgenommen wurden.

Die in diesem Kapitel enthaltenen Bilder und sonstiges Drittmaterial unterliegen ebenfalls der genannten Creative Commons Lizenz, sofern sich aus der Abbildungslegende nichts anderes ergibt. Sofern das betreffende Material nicht unter der genannten Creative Commons Lizenz steht und die betreffende Handlung nicht nach gesetzlichen Vorschriften erlaubt ist, ist auch für die oben aufgeführten nicht-kommerziellen Weiterverwendungen des Materials die Einwilligung des jeweiligen Rechteinhabers einzuholen. 